Margot Werner*

\title{
Work in progress: Die langen Schatten der NS-Vergangenheit
}

\author{
Provenienzforschung und Restitution an der Österreichischen Nationalbibliothek
}

https://doi.org/10.1515/bfp-2020-0003

Zusammenfassung: Die Österreichische Nationalbibliothek hat 2003 ein Projekt zu Erfassung von nach wie vor in ihren Beständen lagerndem Raubgut aus der NS-Zeit initiiert. 52403 Werke wurden nach zweijährigen Recherchen als bedenkliche Erwerbungen eingestuft und werden seither, nach entsprechenden Beschlüssen des österreichischen Kunstrückgabebeirats, an die Erben der Geschädigten übergeben. Die Rückstellungen sind weit fortgeschritten, über 49000 Objekte wurden bereits restituiert.

Daneben ist die Österreichische Nationalbibliothek um größtmögliche Transparenz hinsichtlich ihrer NS-Vergangenheit bemüht: Zwei Ausstellungen und zahlreiche Publikationen zum Thema sowie eine lückenlose ErschlieBung und Öffnung des hauseigenen Archivs zeichnen ein Bild der Vorgänge in der NS-Zeit, der Restitutionen in der Nachkriegszeit sowie der aktuellen Recherchen und geben damit der Öffentlichkeit Einblick in ein dunkles Kapitel der nationalen Gedächtnisinstitution.

Schlüsselwörter: Österreichische Nationalbibliothek; NSZeit; Provenienzforschung; Restitution

\section{Work in Progress: The Long Shadows of the Nazi Past. Provenance Research and Restitution at the Austrian Na- tional Library}

Abstract: In 2003, the Austrian National Library launched a project to identify the stolen goods from the Nazi era still stored in its collections. After two years of research, 52,403 works were classified as questionable acquisitions and have since been handed over to the heirs of the victims in accordance with resolutions of the Austrian Art Restitution Advisory Board. The process of returning the works is well advanced and more than 49,000 items had already been restituted.

In addition, the Austrian National Library endeavours to achieve the greatest possible transparency with regard to its Nazi past: two exhibitions and numerous publications on the subject as well as a complete indexing and

*Kontaktperson: Margot Werner, margot.werner@onb.ac.at opening of the in-house archive present a picture of what happened during the Nazi era, the restitutions in the postwar period and current research, thus giving the public an insight into a dark chapter of the national memory institution.

Keywords: Austrian National Library; NS era; provenance research; restitution

Dieser Beitrag versteht sich als follow-up-Bericht zum vor nunmehr genau 15 Jahren erschienenen Aufsatz „Geraubte Bücher. Die Österreichische Nationalbibliothek stellt sich ihrer NS-Vergangenheit", erschienen in BIBLIOTHEK Forschung und Praxis, 2005 (2) 29, 206-20.

Resümierend soll der lange Weg von der Aufarbeitung der NS-Vergangenheit des Hauses über die Recherche nach geraubten Beständen bis hin zum schon in Sichtweite gerückten Abschluss der Restitution von insgesamt 52403 als bedenkliche Zugänge erfassten Werken geschildert werden. Die lange Zeitspanne der Abwicklung der einzelnen Rückstellungen ist der unglaublich langwierigen Erbensuche geschuldet; erst heuer, im Jahr 2019, ist es z. B. der Israelitischen Kultusgemeinde Wien gelungen, nach fast 15 Jahren die Erbberechtigten nach einem prominentem sozialdemokratischen Politiker ${ }^{1}$ der Zwischenkriegszeit auszuforschen und damit der Österreichischen Nationalbibliothek die Möglichkeit zu geben, ein Konvolut an Druckschriften an diese zu restituieren.

„Damit wird wertvolles deutsches Kulturgut vornehmlich der Ostmark aus jüdischem Besitze in staatlichen übergeleitet und der Allgemeinheit erhalten bleiben. “2

1 Dr. Wilhelm Ellenbogen, ein prominenter Politiker der Zwischenkriegszeit, emigrierte 1938 in die USA und starb dort 1951 kinderlos. Erst 2019 konnte ein österreichischer Kinderhilfe-Verein als erbberechtigt identifiziert werden. Die Restitution wurde umgehend in die Wege geleitet und im Juli 2019 durch Rückgabe und anschließenden Rückkauf von 82 Druckschriften abgeschlossen.

2 Paul Heigl an den RMf. Erziehung, Wissenschaft und Volksbildung im Zuge der Beschlagnahme der Sammlung und -bibliothek des Theaterwissenschaftlers Fritz Brukner, 6.8.1940. ÖNB Archiv Zl. 446/1940. 
Dieser Satz des NS-Generaldirektors Dr. Paul Heigl steht symptomatisch für das Verhalten der Nationalbibliothek in der NS-Zeit und fasst die ,Erwerbungspolitik ' der Jahre 1938 bis 1945 treffend zusammen.

Am 16. März 1938, drei Tage nach dem ,Anschluss', wurde der seit $1923 \mathrm{im}$ Amt befindliche Generaldirektor der Nationalbibliothek, Dr. Josef Bick, verhaftet und seiner Funktion enthoben. Bicks Verhaftung war eine Folge seines politischen Engagements im ständischen Parlament: Er war 1934 zum Präsidenten des Bundeskulturrates und Vizepräsidenten des Bundestages ernannt worden. Das kulturpolitisch wichtige Amt des Generaldirektors der Nationalbibliothek wurde am selben Tag Paul Heigl, einem überzeugten Nationalsozialisten, übertragen. Heigl hatte schon 1933 die Parteimitgliedschaft der NSDAP angenommen und war der SS beigetreten.

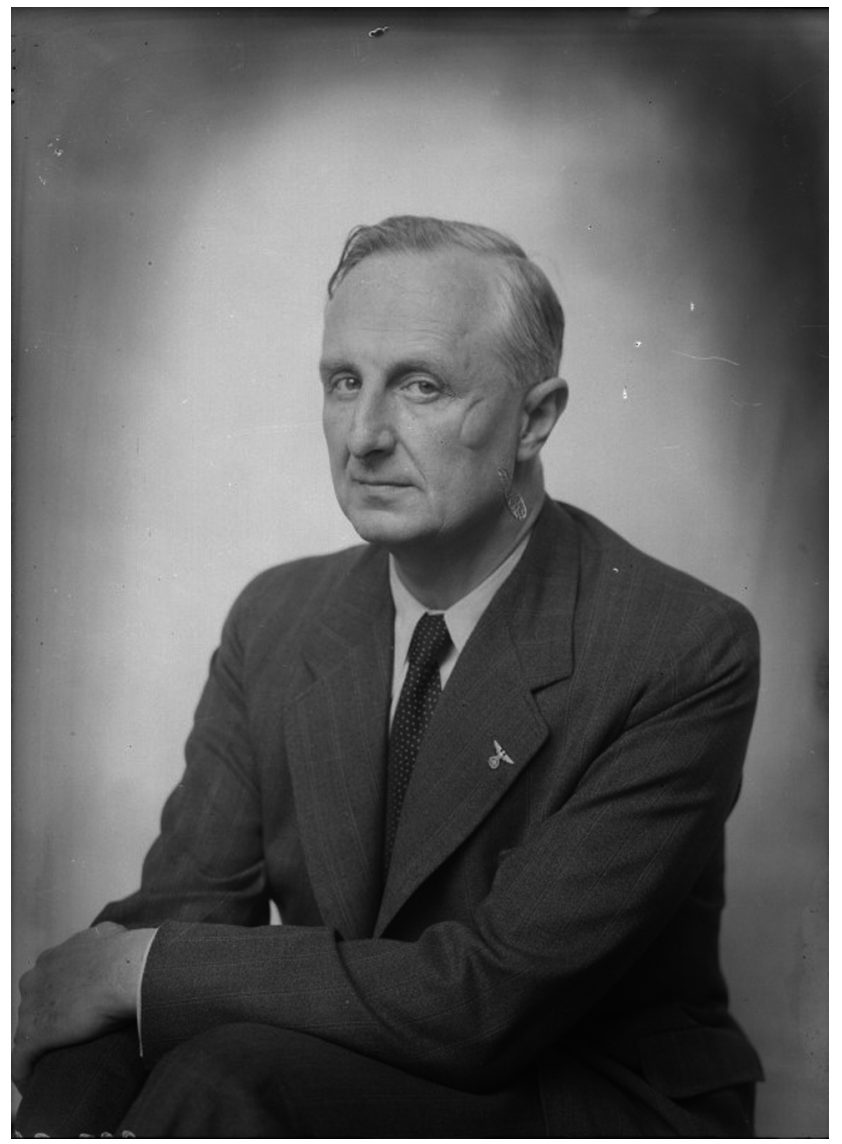

Abb. 1: NS-Generaldirektor Dr. Paul Heigl mit Parteiabzeichen am Revers (das Abzeichen wurde in späteren offiziellen Darstellungen zur Hausgeschichte retuschiert). Heigl beging im April 1945 Selbstmord.

\section{Die Nationalbibliothek in der Kriegszeit}

1938 war die Nationalbibliothek mit einem Bestand von rund 1,3 Mio. Objekten die größte Bibliothek der ,Ostmark ${ }^{*}$ und nach Berlin und München die drittgrößte Bibliothek des Deutschen Reiches. Sie umfasste neben der Druckschriftensammlung acht Sondersammlungen: die Handschriftensammlung, die Papyrussammlung, die Musiksammlung, die Theatersammlung, die Kartensammlung, die Porträtsammlung, das Bildarchiv und eine Orient-Südosteuropa-Abteilung. Letztere nimmt eine Sonderstellung ein, bestand sie doch nur während der NS-Zeit und hatte die Aufgabe, nicht nur bereits im Bestand befindliche Werke unter diesem Themenkomplex zu vereinen, sondern vor allem auch beschlagnahmte Literatur aus dem südosteuropäischen Raum aufzunehmen. Noch heute finden sich Bücher mit dem Kürzel „O.S.“ in den Beständen der Bibliothek. Die Orient-Südosteuropa-Abteilung selbst wurde nach Kriegsende sang- und klanglos wieder aufgelöst. ${ }^{3}$

Mehr schlecht als recht wurde versucht, die Nationalbibliothek auch in der Kriegszeit geöffnet zu halten, Budgetkürzungen, Energiesparmaßnahmen und die Einberufung von Mitarbeitern zum Kriegs- bzw. Arbeitsdienst führten jedoch zu teilweisen Schließungen und Einschränkungen. ${ }^{4}$

Der Personalstand unterlag in der NS-Zeit starken Schwankungen: 89 Personen umfasste der Mitarbeiterstand 1938, zwölf Bibliotheksangehörige wurden schon im April 1938 aus rassischen oder politischen Gründen zwangspensioniert bzw. entlassen und durch NSDAP-Mitglieder ersetzt. ${ }^{5} 1943$ waren bereits 40 Mitarbeiter zum Kriegsdienst eingezogen worden, 1944 mussten schließlich mit der behördlichen Aufforderung, Personal für kriegswichtige Aufgaben zur Verfügung zu stellen, die Handschriften-, Musik-, Theater-, Porträt und Papyrussammlung geschlossen werden. ${ }^{6}$

Als im Sommer 1943 die ersten Luftangriffe auf Orte in der Umgebung Wiens einsetzten, stellte sich auch in der Nationalbibliothek die Frage nach einer Bergung ihrer Bestände. Es erwies sich aber aufgrund mangelnder Transportmittel als unmöglich, die gesamte Druckschriftensammlung an einen sicheren Ort zu bringen. Bereits seit Kriegsbeginn waren die kostbarsten Objekte in 90 Kisten verpackt in eigenen Schutzräumen in den Kellern der Na-

3 Trenkler (1973) 128f.

4 Hall und Köstner (2006) $418 \mathrm{ff}$.

5 Trenkler (1973) 102f.

6 Hall und Köstner (2006) 409. 
tionalbibliothek gesichert worden. 1943 entschloss sich Generaldirektor Heigl die Bücher des Prunksaals (200 000 Bände, darunter die Bibliotheca Eugeniana) und die wertvollsten Stücke aus den Sammlungen in den Kellerräumen unter dem Prunksaal einzulagern. ${ }^{7}$ Erst Ende Jänner 1945 wurden schließlich die wertvollsten Handschriften und Druckwerke nach Lauffen bei Bad Ischl gebracht, die Bestände der Porträtsammlung kamen nach Pulkau in Niederösterreich.

Die Nationalbibliothek selbst wurde im Gegensatz zu umliegenden Gebäuden wie die Staatsoper und die Albertina nicht direkt von Bomben getroffen, erlitt jedoch durch den verheerenden Bombenangriff vom 12. März 1945, bei dem auch die Albertina schwere Schäden davontrug, geringe Verluste am Bestand der Musiksammlung, des Bildarchivs und der Handbibliothek der Papyrussammlung. ${ }^{8}$ Ebenso hatte die Bibliothek Verluste durch Plünderungen bzw. die Nichteinbringlichkeit von an ehemalige NS-Behörden verliehene Werken zu verzeichnen. ${ }^{9}$

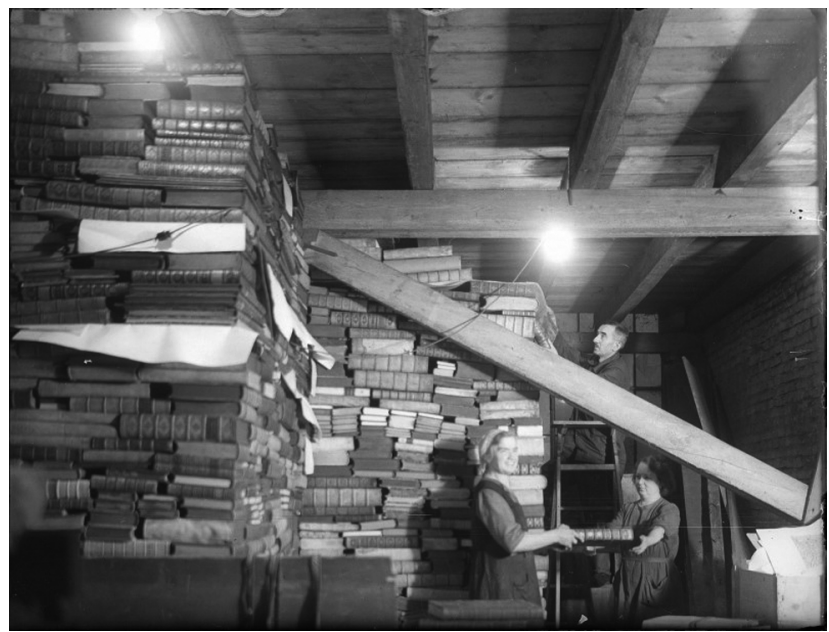

Abb. 2: Bergung der Prunksaal-Bestände, 1943

\section{Institutionalisierter Raub}

Mit Kriegsbeginn waren die Möglichkeiten des Handels und Tausches mit dem Ausland weitgehend unterbrochen, diese Lücke versuchte Heigl durch die Aufnahme von beschlagnahmten Werken zu kompensieren. In Zahlen ausgedrückt bedeutet dies, dass von 70000 in der NS-Zeit für Druckschriften vergebenen Signaturen, über 10000 mit be- schlagnahmten Bänden gefüllt wurden. In diesen Zahlen noch nicht inbegriffen ist die heute nicht mehr schätzbare, jedoch aller Wahrscheinlichkeit nach wesentlich höhere Zahl an entzogenen Bänden, für welche die Nationalbibliothek nur eine Durchgangsstation war: Aus Zeit- und Personalmangel wurde ein großer Teil der beschlagnahmten Bibliotheken nicht einsigniert, sondern verkauft, getauscht oder im Aufbau befindlichen Bibliotheken des Deutschen Reiches zugewiesen. Den Umfang der Beschlagnahmungen verdeutlicht eine Korrespondenz Heigls aus dem Jahr 1940: Er bittet das Reichsfinanzministerium um Zuteilung weiterer Bücherregale, da „fast 300000 Bände in den Magazinen lagern und aufgearbeitet werden müssen und zweifellos noch viel Material hereinkommen wird. " ${ }^{10}$

Heigls gute Beziehungen zu Gestapo, SS und SD waren zweifellos seiner offensiven Erwerbungspolitik in den folgenden Jahren dienlich. Im Aktenbestand der Bibliothek sind zahlreiche Ansuchen Heigls an die für Beschlagnahme und Verwertung jüdischen Vermögens zuständigen Stellen erhalten.

Bereits kurz nach dem ,Anschluss' wurden der Nationalbibliothek ,sichergestellte' Bibliotheken und Sammlungen vorerst zur Verwahrung, nach Klärung der Eigentumsfrage - das heißt Beschlagnahme oder Verfall - zur Aufnahme in ihre Bestände übergeben. Allerdings erfolgte nicht in allen Fällen eine der NS-Gesetzgebung konforme Eigentumsübertragung. Vielfach verblieben die Objekte lediglich zur Verwahrung im Haus. Für die Vorbesitzer änderte diese Tatsache wenig: Sowohl ,sichergestellte' als auch definitiv verfallene Objekte mussten nach Kriegsende mühsam ausgeforscht werden.

Wie viele geraubte Bücher und Sammlungsobjekte in der NS-Zeit insgesamt in die Nationalbibliothek eingebracht wurden, ist heute nicht mehr mit Sicherheit zu beantworten: Allein jene Fälle namentlich bekannter vormaliger Eigentümer umfassen aber mindestens 150000 Druckschriften und rund 45000 Sammlungsobjekte. Rechnet man nun die anonym von der Gestapo eingebrachten Objekte hinzu, so erhöht sich diese Zahl - vorsichtig geschätzt - auf das Doppelte. Das heißt also, es ist zwischen 1938 und 1945 von einem Zuwachs von 400000 bis 500000 beschlagnahmten Objekten auszugehen.

10 Heigl an Baccarcich (Reichsfinanzministerium) v. 4. August 1940, ÖNB Archiv, ohne Zl., Mappe „Bibliothek der Reichsstatthalterei“.
7 Trenkler (1973) 114.

8 Trenkler (1973) 122.

9 ÖNB Archiv, Zl. 732/1946. 


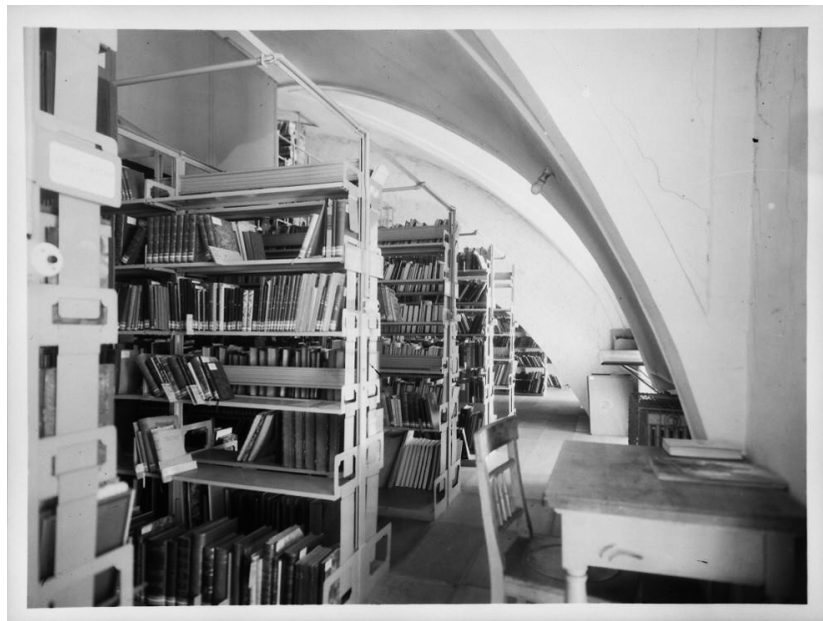

Abb. 3: Beschlagnahmte Signaturengruppe, 1944

\section{Die Geschädigten}

Hinsichtlich der Herkunft der geraubten Bibliotheken sind mehrere Gruppen von Geschädigten zu unterscheiden: An erster Stelle waren jüdische Privatpersonen und Institutionen, wie zum Beispiel die Israelitische Kultusgemeinde Wien und die ihr angeschlossenen Landesgemeinden, von der Beschlagnahme ihrer Bibliotheken und Sammlungen betroffen. $\mathrm{Zu}$ nennen sind ebenso große Privatbibliotheken und Sammlungen jüdischer Verfolgter, darunter die Bibliothek Oskar und Gerhard Ladners im Umfang von ca. 5000 Bänden, die Bibliothek Harald Reininghaus/Stefan Auspitz im Umfang von ca. 4800 Bänden, die Bibliothek und Exlibrissammlung Marco Birnholz von ca. 1500 Bänden und 30000 Exlibris, die große theatergeschichtliche Sammlung und Bibliothek Fritz Brukners, die über 6000 Bände umfassende Notensammlung des Gottlieb Kaldeck, die Bibliothek Heinrich Schnitzlers von etwa 6000 Bänden, 3000 Bände und eine Manuskriptesammlung von Norbert Jokl, mehre tausend Bände aus dem Besitz Moritz Kuffners und die umfassende Privatbibliothek von Alphonse de Rothschild.

Neben den klingenden Namen sollen aber auch die beschlagnahmten Büchermassen anonymer Herkunft nicht in Vergessenheit geraten. Betroffen waren vor allem kleine Privatbibliotheken ausgewanderter oder deportierter Verfolgter, die in deren Wohnungen zurückgelassen worden waren und zumeist über die Gestapo in die Nationalbibliothek eingewiesen wurden. Von Beschlagnahme und Vermögensentzug betroffen waren weiters der nationalsozialistischen Ideologie entgegenstehende Vereine und politische Organisationen, wie zum Beispiel die Freimaurerlogen und die Bibliothek des Psychoanalytischen Ver- lags. Gemäß der Verordnung über die Einziehung volksund staatsfeindlichen Vermögens im Lande Österreich (18.11.1938) wurden auch die Bibliotheken von kulturellen Institutionen und staatlichen Einrichtungen der vom Deutschen Reich besetzten Gebiete beschlagnahmt und eingezogen. Der diesbezüglich umfangreichste Bestand der in die Nationalbibliothek eingeliefert wurde, war die über 120000 Bände umfassende Bibliothek des Wiener Komensky-Schulvereins. Ebenfalls der Nationalbibliothek zugewiesen wurden die Bibliotheken tschechischer Schulen und jene der Tschechischen Kulturhistorischen Kommission in Wien. ${ }^{11}$ Nach der Besetzung der Tschechoslowakei, Polens und Jugoslawiens wurden die Bibliotheken der Konsulate dieser Staaten ebenfalls als feindliches Vermögen beschlagnahmt und in Folge von der Nationalbibliothek übernommen.

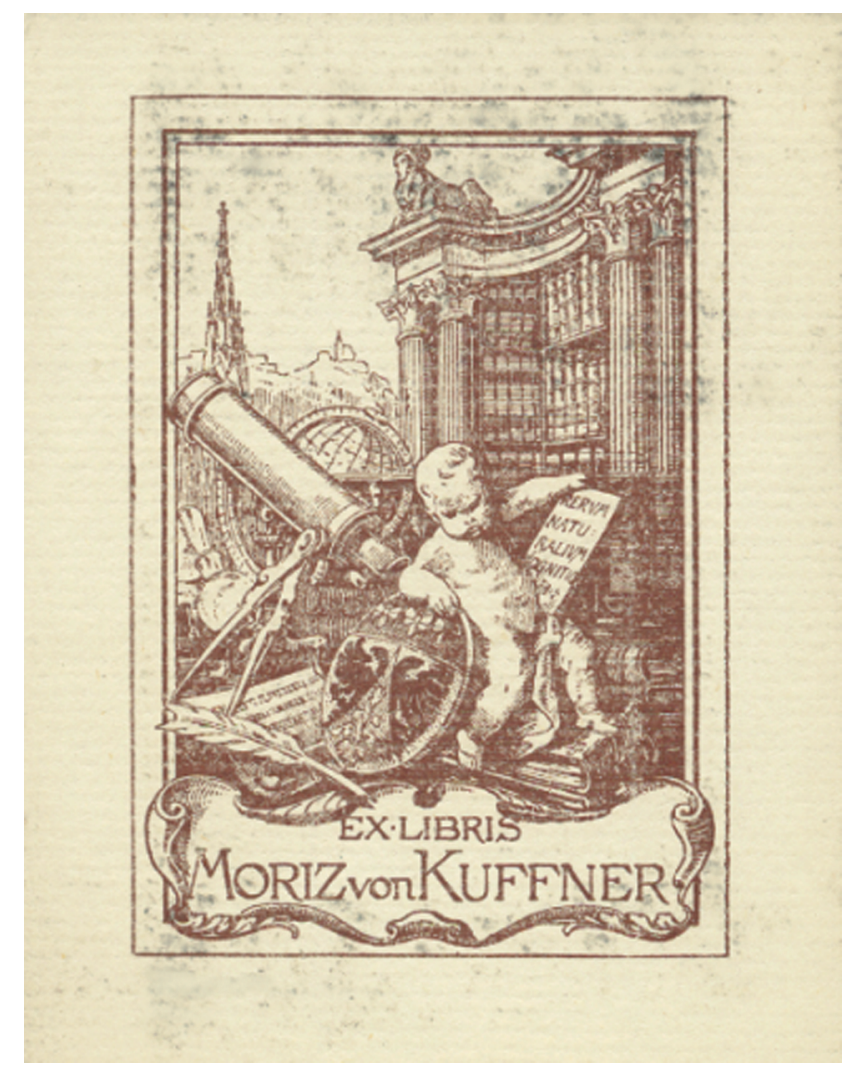

Abb. 4: Exlibris des verfolgten Moritz von Kuffners, damaliger Inhaber der Ottakringer Brauerei und Errichter der heute noch bestehenden Kuffner-Sternwarte

11 ÖNB-Archiv-Mappe Tschechisches Vermögen. 


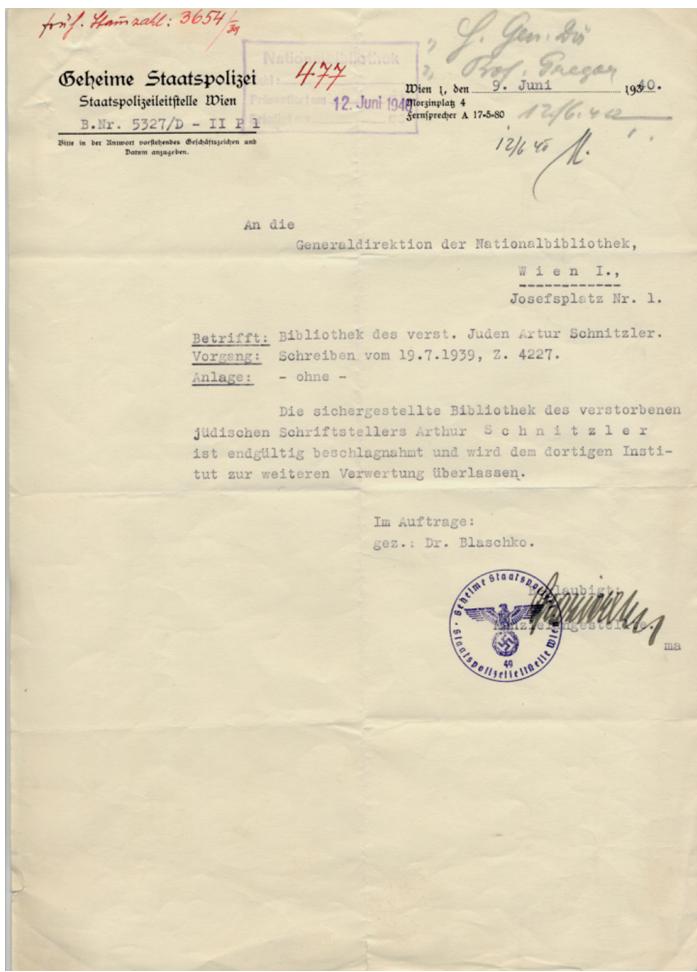

Abb. 5: Die Gestapo informiert die Nationalbibliothek über die Zuweisung der zuvor von dieser urgierten beschlagnahmten Bibliothek Arthur und Heinrich Schnitzlers

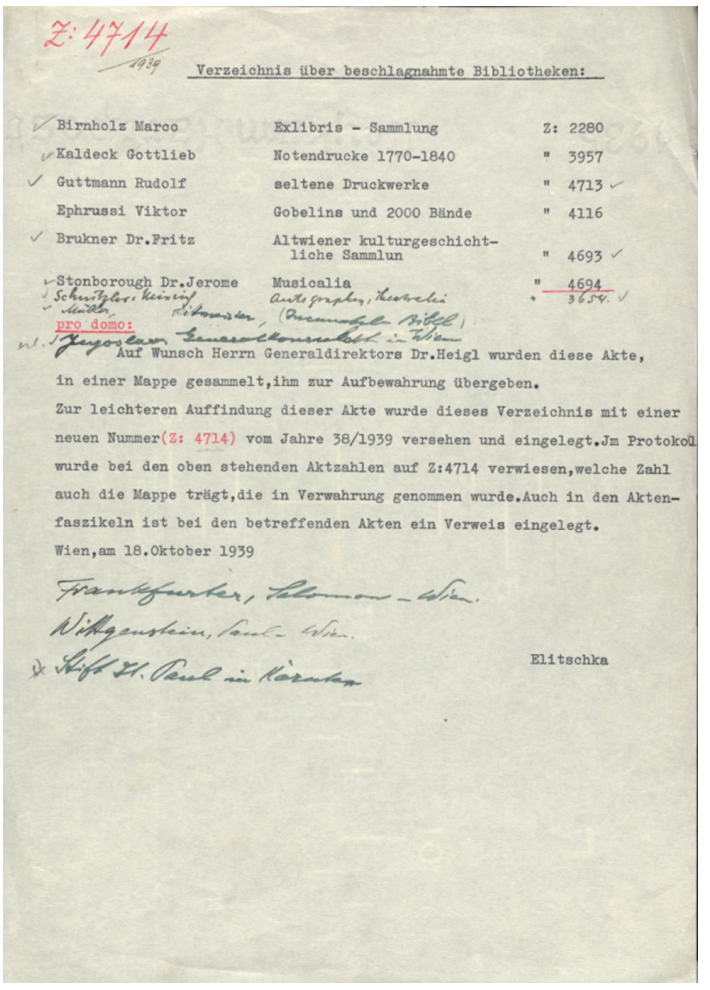

Abb. 6: Das einzige im ÖNB-Archiv aus der NS-Zeit erhalten gebliebene Verzeichnis über beschlagnahmte Bibliotheken

\section{Der Umgang mit dem NS-Erbe in der Nachkriegszeit}

Bereits im Oktober 1945 wurde der Lesebetrieb wieder aufgenommen. Die in den Kellerräumen gelagerten Bestände wurden im Sommer 1945 wieder aufgestellt und die in den Bergungsorten außerhalb Wiens gelagerten Werke wurden 1946 nach Wien gebracht.

Nach Kriegsende hatte die Nationalbibliothek großen Personalschwund zu verzeichnen: 1944/45 umfasste der Personalstand rund 150 Mitarbeiter. Davon wurden 34 als Nationalsozialisten sofort entlassen. 20 Mitarbeiter schieden aus diversen anderen Gründen, etwa weil sie Reichsdeutsche oder nur auf Kriegsdauer beschäftigt waren, aus. Im Rahmen der vom Staatsamt für Volksaufklärung eingerichteten Sonderkommission für Entnazifizierung im öffentlichen Dienst wurden weitere 16 Mitarbeiter geprüft, die erst nach März 1938 Mitglied der NSDAP geworden waren, acht dieser Personen mussten als „politisch belastet“ die Dienststelle verlassen. ${ }^{12}$

Darüber hinaus hatte die Nationalbibliothek mit elf Gefallenen einen hohen Verlust erlitten.

Die Rückgabe entzogener Bibliotheken war nicht nur aufgrund des Personalmangels mit großen Schwierigkeiten verbunden: Jene umfangreichen Bibliotheken bekannter Sammler und Institutionen, die noch als Einheit in den Magazinen deponiert waren, konnten relativ leicht festgestellt und restituiert werden. Ebenso wurden die erwähnten Bibliotheken der ausländischen Gesandtschaften sowie jene der kulturellen Institutionen der ehemals besetzen Gebiete bald nach Kriegsende zurückgestellt.

Problematisch gestaltete sich die Suche im Fall der aussortierten und zur weiteren Verwertung bestimmten Dubletten sowie hinsichtlich der inventarisierten Bestände. Die Österreichische Nationalbibliothek ${ }^{13}$ meldete zwar einen Großteil der bekannten übernommenen Bibliotheken gemäß der Vermögensentziehungs-Anmeldungsverordnung bei den zuständigen Bezirksverwaltungsbehörden an, konnte aber in den wenigsten Fällen Umfang und Inhalt näher präzisieren. Obwohl die Ausforschungsarbeiten seitens des damit beauftragten Beamten Ernst Trenkler engagiert betrieben wurden, mussten sich die Geschädigten mit dem Verlust von erheblichen Teilen ihrer Sammlungen abfinden. Die Rückgabe erfolgte zumeist gemäß dem 1. Rückstellungsgesetz von 1946 über einen Bescheid

12 Hall und Köstner (2006) 479 ff.

131945 Umbenennung in „Österreichische Nationalbibliothek“. 
der Finanzlandesdirektion. ${ }^{14}$ 1947-49 wurde zur Ermittlung der einsignierten entzogenen Druckschriften zwar eine Buchautopsie in den Magazinen durchgeführt, jedoch aller Wahrscheinlichkeit nach nur innerhalb jener Signaturengruppe, die zur Gänze für enteignete Bücher vorgesehen war. ${ }^{15}$

Insgesamt restituierte die Österreichische Nationalbibliothek aus den inventarisierten beschlagnahmten Buchbeständen circa 5420 Titel. In Relation zur Gesamtzahl der entzogenen, in den Bestand aufgenommenen Werke, wurde also nur etwa ein Drittel im Zuge der Nachkriegsrestitutionen wieder ausgeschieden. Generell war die Restitution in der Nachkriegszeit auf Geschädigte, die aktiv ihren Rückstellungsanspruch geltend machten, beschränkt. Hinsichtlich der zahlreichen einsignierten Werke unbekannter Vorbesitzer, konnte in der Nachkriegszeit keine Lösung gefunden werden.

\section{Provenienzforschung und Rückstellungsbemühungen der Gegenwart}

Im Jahr 1950 waren die Restitutionen aus der Österreichischen Nationalbibliothek eingestellt worden - ungeachtet der Tatsache, dass noch zahlreiche unrechtmäßig erworbene Bücher und auch Sammlungsobjekte in den Magazinen lagerten. Erst mit Erlass des Österreichischen Kunstrückgabegesetzes (BGBl. 181/1998) wurde das Thema der unerledigten Restitutionen wieder aufgegriffen und auf Initiative der 2001 bestellten Generaldirektorin Dr. Johanna Rachinger im Jahr 2002 ein Projekt zur Erfassung von NS-Raubgut initiiert.

Um einen Eindruck von der Größenordnung des in den Jahren 2002-2003 an der Österreichischen Nationalbibliothek durchgeführten Erfassungsprojekts zu vermitteln, scheint es geboten, zu allererst die Rechercheergebnisse zu nennen: 52403 Einzelobjekte - Bücher, Fotos, Negative, Autografen, Handschriften, Karten und Musikalien mussten nach Abschluss der Erhebungsarbeiten als bedenkliche Erwerbungen der NS-Zeit, oder deutlicher gesagt, als gestohlen, eingestuft werden.

14 Rückgestellt wurden die Bibliotheken und Sammlungen von Harald Reininghaus (Vorbesitz Stefan Auspitz), Viktor von Ephrussi, Großloge Wien, Komensky-Schulverein, Moritz von Kuffner, Alphonse de Rothschild, Heinrich Schnitzler, Fritz Brukner, Gottlieb Kaldeck und Marco Birnholz.

15 GD Stummvoll an Huber (Sammlung Brukner) vom 1.3.1954, ÖNB Archiv, Zl. 107/1954.
Im Zuge des Projekts wurde nachgeholt, was in der Nachkriegszeit verabsäumt wurde: Eine Generalautopsie aller fraglichen Bestände: rund 200000 Objekte - 150000 Bände Druckschriften und 50000 Sammlungsobjekte wurden einer Überprüfung unterzogen. Im Gegensatz zu Werken der bildenden Kunst müssen Provenienzforscher in Bibliotheken auf Hilfsmittel wie Werkverzeichnisse, Auktionskataloge und Objektbeschreibungen in der Literatur verzichten. Bibliotheken sind bei der Recherche nach der Herkunft eines Objekts also auf hausinterne Inventare, Eingangsbücher und Archivalien angewiesen. Als unschätzbare Quelle erwies sich dabei das weitgehend vollständig erhaltene Archiv der Generaldirektion. Zuweisungen gröBerer und wertvoller Bibliotheken und Sammlungen sind gut dokumentiert. Schlecht bis überhaupt nicht dokumentiert ist hingegen die Zulieferung jener Unzahl an Druckschriften und Sammlungsobjekten, die aus kleinen Sammlungen vertriebener oder deportierter Verfolgter stammt.

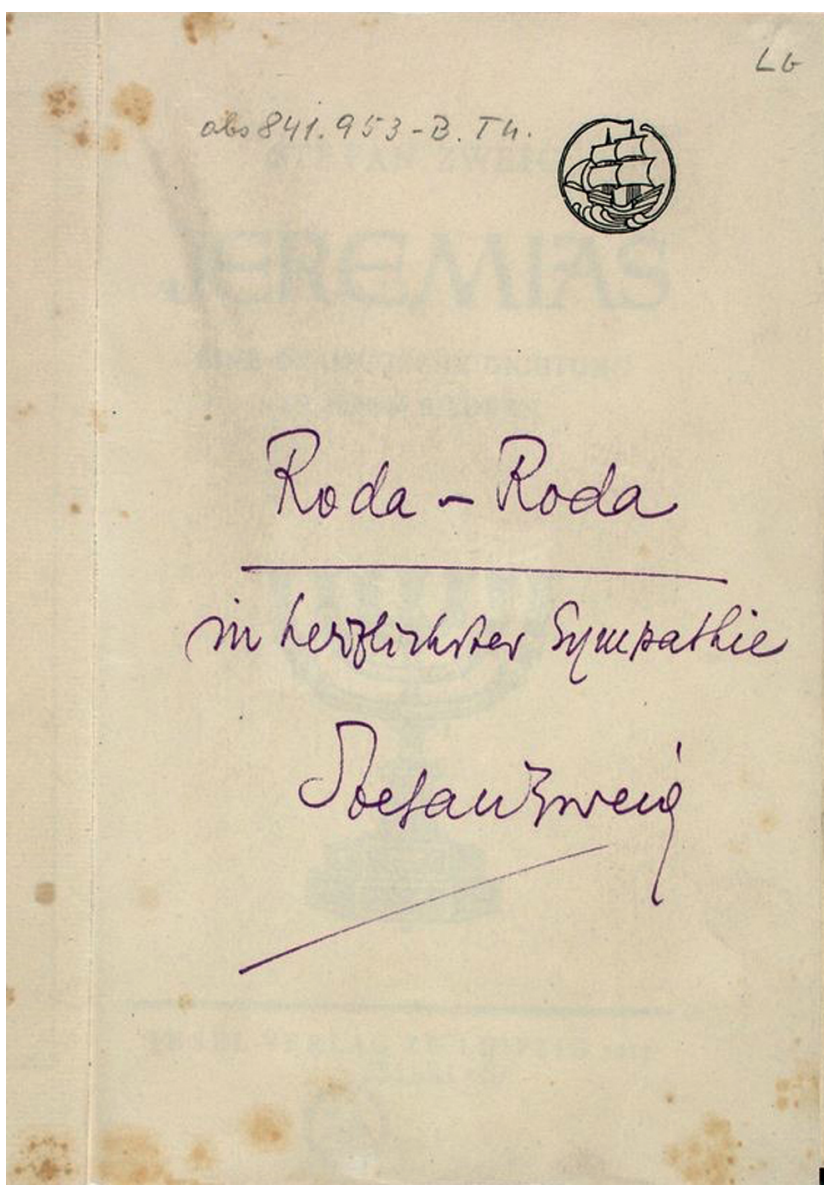

Abb. 7: Aus der beschlagnahmten Bibliothek Roda Rodas: Stefan Zweig, Jeremias, Leipzig 1917, eigenhändige Widmung an Roda Roda (Alexander Friedrich Rosenfeld) 


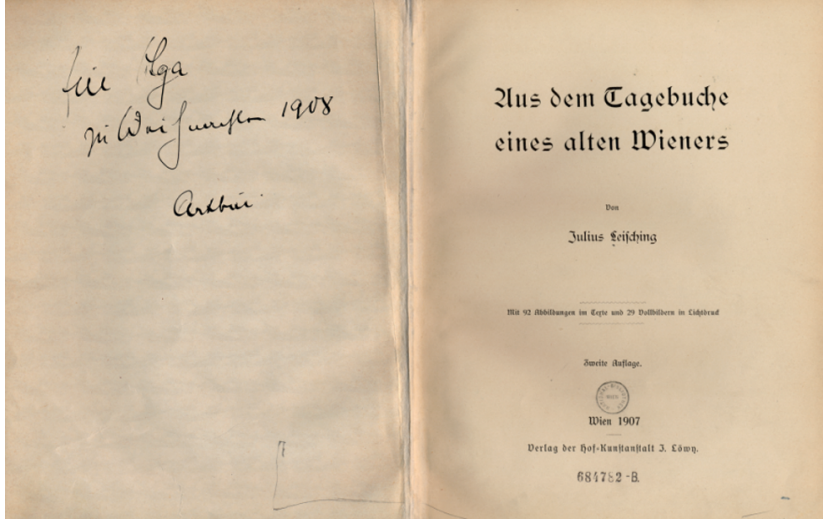

Abb. 8: Aus der beschlagnahmten Bibliothek Heinrich Schnitzlers: Widmung seines Vaters Arthur Schnitzler an dessen Frau Olga

Die im Zuge der Autopsie vorgefundenen Merkmale waren vielfältig: Leider fanden sich in den wenigsten Fällen Exlibri oder Namensstempel, häufiger handschriftliche Besitzvermerke oder Notationen, teilweise auch markante Neubindungen, die die gemeinsame Herkunft mehrerer Bücher aus einer Bibliothek vermuten ließen. Die Mehrzahl der anonymen, von der Gestapo formlos abgelieferten Bücher bleibt aber, sofern sie keinen Besitzvermerk oder ein besonderes Merkmal aufweisen, zwangsläufig erblos.

Die Österreichische Nationalbibliothek war allerdings aufgrund eines besonderen Merkmals zumindest in der Lage, beschlagnahmte Bücher, unabhängig davon, ob sie einen Vorbesitzervermerk trugen oder nicht, zu identifizieren: Jene beschlagnahmten Objekte, die durch Inventarisierung Aufnahme in die Bestände des Hauses fanden, wurden mit einem markanten Kürzel gekennzeichnet, das die heutigen Forscher erst in die Lage versetzt, beschlagnahmtes von rechtmäßig erworbenem Eigentum zu unterscheiden. Als Provenienzangabe, die geeignet war, die Herkunft der Bestände zu verschleiern - denn das war die Vorgabe der damaligen Leitung ${ }^{16}$-, wurde die Sigle $P 38$ als Abkürzung für Polizei 1938 gewählt. Dieses Kürzel wurde sowohl in die beschlagnahmten Bücher selbst, als auch in die jeweiligen Inventare als Provenienzangabe eingetragen.
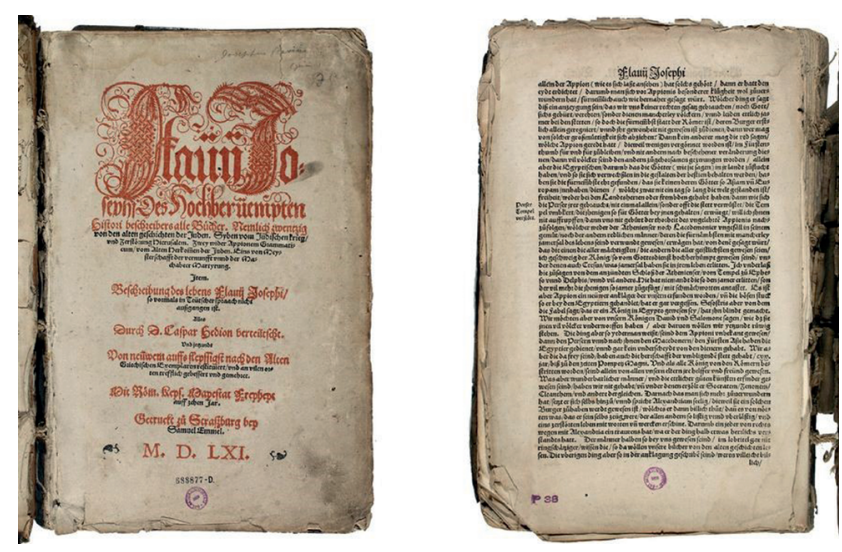

Abb. 9: Stempel „P (-olizei 19)38“ in der Druckschrift „)osephus Flavius (germ.), Flavii Josephi des Hochberuempten Histori beschreibers alle Bücher, nemlich zwenzig, Straßburg 1561“

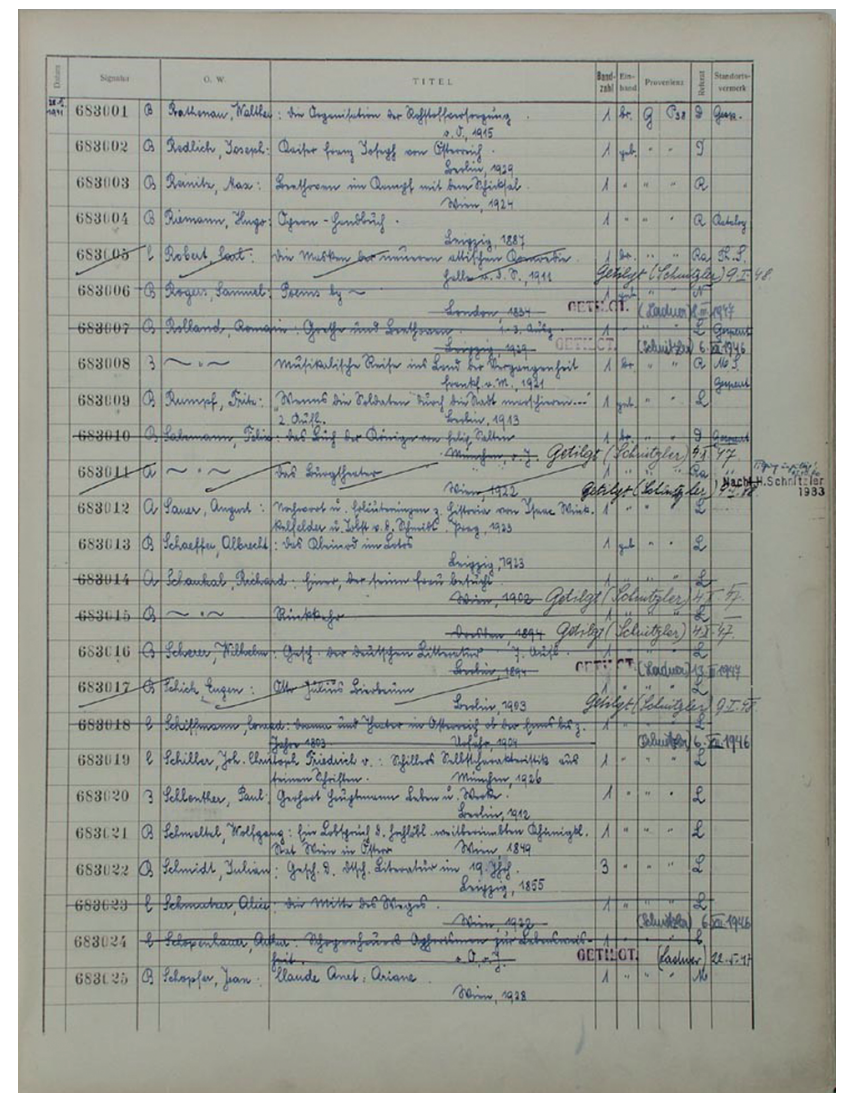

Abb. 10: Für beschlagnahmte Zuwächse („Provenienz P 38“) reserviertes Inventar der Druckschriftensammlung 


\section{Aktueller Stand der Rückstellungen}

Als Gesamtergebnis der Forschungen in allen Abteilungen des Hauses lag nicht nur eine Liste der erwähnten 52403 vermutlich entzogenen Objekte, sondern auch ein Verzeichnis von etwa 800 verschiedenen Eigentumszeichen von Privatpersonen und Institutionen vor, das Grundlage der weiteren Recherchen war. In 72 Fällen $^{17}$ ist es gelungen, die Entziehungsgeschichte und die damit verbundenen Schicksale der Verfolgten zu recherchieren. Jene Eigentumsvermerke, die nicht identifiziert werden konnten, da sie zum Teil nur aus Namensfragmenten bestehen, wurden vom Nationalfonds der Republik Österreich für Opfer des Nationalsozialismus in einer Online-Kunstdatenbank publiziert, um vormaligen Eigentümer bzw. deren Erben die Möglichkeit zur Anspruchserhebung zu geben.

Die Österreichische Nationalbibliothek hat sich mit tatkräftiger Unterstützung der Israelitischen Kultusgemeinde Wien und des Nationalfonds zudem der sehr schwierigen Aufgabe der Suche nach den Erbberechtigten gestellt. Mit teils enormem Recherche- und Kommunikationsaufwand wurde und wird versucht, Nachkommen der Verfolgten auf der ganzen Welt ausfindig zu machen und zu kontaktieren. Es ist bislang gelungen, 70 Einzelfälle abzuwickeln und zusammen 49204 Objekte an ihre rechtmäßigen Eigentümer zu restituieren (Stand Oktober 2019). Darunter befinden sich auch 8363 Bücher die im Jahr 2010 und 2255 Bücher die im Jahr 2018 mangels Vorbesitzerhinweisen an den laut Österreichischem Kunstrückgabegesetz (BGBl. 181/1998) empfangsberechtigten Nationalfonds der Republik Österreich für Opfer des Nationalsozialismus restituiert wurden. Beide Tranchen an erblosen Werken wurden auf Wunsch des Nationalfonds von der Österreichischen Nationalbibliothek zurückgekauft. Die Österreichische Nationalbibliothek ist damit ihrem Ziel, der vollständigen Rückgabe aller noch im Haus befindlichen geraubten Objekte, sehr nahe.

Der verbleibende Rest an noch nicht restituierten Büchern und Sammlungsobjekten wird bis Ende 2020 einer nochmaligen Recherche unterzogen, um zu prüfen, ob mittels neuer Quellen für einige der Werke doch noch Vorbesitzer ermittelt werden können. Es handelt sich um rund 800 bislang unauflösbare Besitzerhinweise (unvollständi-

17 Diese 72 Fälle umfassen ca. 3/4 der ausgeforschten beschlagnahmten Werke mit Namenshinweis. Bei den übrigen Namenshinweisen ist meist nur ein Buch betroffen und die Aktenlage entsprechend dürftig. ge Namen, Initialen, Besitzstempel etc.), denen im Rahmen zweier Projekte erneut nachgegangen wurde bzw. wird.

Die Themen Provenienzforschung und Restitution nehmen auch abseits der konkreten Recherchen nach wie vor einen hohen Stellenwert in der Österreichischen Nationalbibliothek ein. Um der interessierten Öffentlichkeit das Schicksal Verfolgter, das sich hinter anonym eingelieferten Büchermassen verbirgt, vor Augen zu führen und mit dem Anspruch eines offenen Umgangs mit der NS-Vergangenheit des Haues, initiierte die Österreichische Nationalbibliothek die bis dahin erste Ausstellung zum Thema Bücherraub: Unter dem Titel Geraubte Bücher. Die Österreichische Nationalbibliothek stellt sich ihrer NS-Vergangenheit präsentierte das Haus 2004 eine publikumswirksame Zusammenstellung der Ergebnisse der Provenienzforschung.

2006 legten Murray G. Hall und Christina Köstner ein von Generaldirektorin Rachinger unterstütztes umfassendes Werk über die Geschichte der Nationalbibliothek in der NS-Zeit mit dem Titel ... , allerlei für die Nationalbibliothek $z u$ ergattern.... Eine österreichische Institution in der NSZeit vor und 2008 widmete die Österreichische Nationalbibliothek anlässlich der Restitution einer über 20000 Fotos umfassenden Sammlung dem Thema NS-Raub erneut eine Ausstellung. Unter dem Titel Zur Erinnerung an schönere Zeiten. Bilder aus der versunkenen Welt des jüdischen Sammlers Raoul Korty wurde anhand von persönlichen Dokumenten und der großen Fotosammlung dem Schicksal des 194. ermordeten Sammlers gedacht.

Nicht zuletzt ist zu erwähnen, dass Generaldirektorin Rachinger unmittelbar nach Vorlage des Provenienzberichts im Jänner 2004 den Auftrag zur Erschließung des Archivs der Österreichischen Nationalbibliothek erteilte. Alle historischen Akten, insbesondere aber der Aktenbestand zur NS-Zeit, sollten im Sinne der größtmöglichen Transparenz Forscher zugänglich gemacht werden. Das Projekt ist mittlerweile abgeschlossen, alle Verwaltungsakten der NS-Zeit, inklusive dem Bestand zu Vermögensentzug und Restitution, stehen detailliert erschlossen und mit Inhaltsangaben versehen über den Online-Katalog QuickSearch der Österreichischen Nationalbibliothek zur Verfügung.

\section{Abbildungsverzeichnis}

Abb. 1: ÖNB, Bildarchiv, 237.913-B

Abb. 2: ÖNB, Bildarchiv, 237918-B

Abb. 3: ÖNB, Bildarchiv, Bestand Reiffenstein, 106.340

Abb. 4: ÖNB, Scan aus beschlagnahmtem Werk

Abb. 5: ÖNB, Archiv, Zl. 477/1940 
Abb. 6: ÖNB, Archiv, Zl. 4714/1939

Abb. 7: ÖNB, Sig. 231.881-B Neu Mag (bereits restituiert)

Abb. 8: ÖNB, Sig. 684.782-B Neu Mag (bereits restituiert)

Abb. 9: ÖNB, Sig. 688.877-D Alt Mag

Abb. 10: ÖNB, Druckschriften-Inventar Sig. 680.000 bis

690.000

alle@ ÖNB

\section{Literaturverzeichnis}

Trenkler, Ernst (1973): Geschichte der Österreichischen Nationalbibliothek. Zweiter Teil. Die Nationalbibliothek (1923-1967), hg. v. Josef Stummvoll und Rudolf Fiedler, Wien.

Hall, Murray G.; Köstner, Christina (2006): „,...allerlei für die Nationalbibliothek zu ergattern...“. Eine österreichische Institution in der NS-Zeit, Wien.

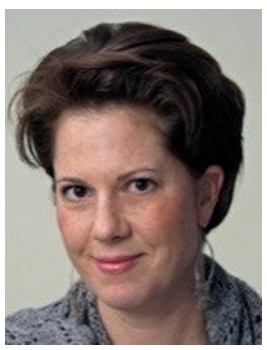

\section{Margot Werner}

Österreichische Nationalbibliothek

Josefsplatz 1

A-1015 Wien

Wien

margot.werner@onb.ac.at 